\title{
The value of the impact factor should not be underestimated
}

\author{
Yoshiki Hirooka ${ }^{1}$
}

Received: 20 June 2021 / Accepted: 25 June 2021

(c) The Japan Society of Ultrasonics in Medicine 2021

The third state of emergency will be lifted today (June 20) in Japan, with the exception of Okinawa Prefecture. According to an announcement by the government, the Novel Coronavirus Response Headquarters officially decided on June 17 to lift the state of emergency in nine administrative divisions (Hokkaido, Tokyo, Aichi, Osaka, Kyoto, Hyogo, Okayama, Hiroshima, and Fukuoka) on June 20. However, the state of emergency will be extended until July 11 only in Okinawa Prefecture. Of the nine administrative divisions where it will be lifted, seven administrative divisions (Hokkaido, Tokyo, Aichi, Osaka, Kyoto, Hyogo, and Fukuoka) will shift to semi-state of emergency coronavirus measures. The time limit will be until July 11 along with Saitama Prefecture, Kanagawa Prefecture, and Chiba Prefecture, where semi-state of emergency coronavirus measures are already in effect.

The Tokyo 2020 Olympics are scheduled to be begin on July 23. The world will have its eyes on the outcome of this summer's Olympics, which will be held amidst the ongoing novel coronavirus epidemic. We can only pray that the Olympics will not lead to a fifth wave of novel coronavirus infections. I think that many of our readers are healthcare professionals. I commend the courage and efforts of healthcare professionals confronting the novel coronavirus infection around the world, and I pray for their safety.

Changing topics, 2020 impact factors will be announced later than ordinary on June 30. The latest impact factor for Japan Society of Ultrasonics in Medicine's English journal, Journal of Medical Ultrasonics (JMU), is forecast to be 1.264. It will be the first time that the impact factor for JMU will exceed 1.0. As Editor-in-Chief of JMU, I would like to sincerely thank the members of the Editorial Board for all their help. Simply put, the impact factor is akin to the rank of a journal. Other indices are also important—such as the citation index, which one could say is the rank of the articles themselves, and the h-index, which indicates the relative contribution of a scientist's research based on the number of papers and the number of citations-but I think that the impact factor is generally the best-known index. The Editorial Board is happy that the impact factor will exceed 1.0 for the first time. We intend to sustain our efforts, so that the impact factor ascends even higher in 2021.

The Editorial Board has come up with a variety of strategies over the years to raise JMU's impact factor and its international profile. One such strategy has been to publish features that appeal to our readers in disciplines that deal with ultrasound. JMU is fundamentally a journal that publishes papers on basic medicine and clinical medicine as they relate to ultrasound, but features are put together in a way that makes them appealing, such as allowing the inclusion of CT or MRI reviews and pathology reviews as appropriate, so that readers can grasp the full picture of a condition by becoming acquainted with the relationship between ultrasound and other modalities or histopathology. We publish features that allow an author writing a paper in a certain domain to get all the information they need by simply reading JMU's feature. We publish features that provide an understanding of the full picture of a domain and that include all of the necessary references for those carrying out research and those writing papers on ultrasound not only in clinical medicine domains but also in basic medicine domains. JMU's Editorial Board is comprised of a variety of experts in domains such as basic engineering and clinical medicine. We plan to continue to publish many features that

Yoshiki Hirooka

hirooka@med.nagoya-u.ac.jp

1 Department of Gastroenterology and Hepatology, Fujita

Health University, Aichi, Japan 
will benefit our readers, covering not only a wide range of the most up-to-date topics but also topics that will provide an understanding of the basics. The Editorial Board hopes to mobilize all of its energy to produce a JMU that receives wide recognition from researchers from all over the world involved in medical ultrasonics.

Publisher's Note Springer Nature remains neutral with regard to jurisdictional claims in published maps and institutional affiliations. 